\title{
Estudo dos efeitos da consideração de cargas dependentes da frequência no Modelo de Sensibilidade de Potência
}

\author{
André Luiz Miyahara Takahashi ${ }^{1}$ \\ Faculdade de Engenharia de Ilha Solteira, UNESP, Ilha Solteira, SP \\ Percival Bueno Araujo ${ }^{2}$
}

Faculdade de Engenharia de Ilha Solteira, Departamento de Engenharia Elétrica, UNESP, Ilha Solteira, SP

\begin{abstract}
Resumo. Neste trabalho são apresentados resultados obtidos com a inclusão de modelos dinâmicos para representação de cargas em sistemas elétricos por meio do Modelo de Sensibilidade de Potência. Dos resultados obtidos concluiu-se que tal representação, em pequena escala, produz resultados relativamente semelhantes aos obtidos quando utilizados modelos estáticos para a representação de cargas.

Palavras-chave. Sistemas multimáquinas, estabilidade a pequenos sinais, Modelo de Sensibilidade de Potência, cargas não lineares, análise no Espaço de Estado
\end{abstract}

\section{Introdução}

Um dos maiores desafios na análise de problemas envolvendo sistemas de energia é a escolha de um modelo adequado. Enquanto que modelos muito simples acabam por não representar adequadamente o sistema, por outro lado, modelos muito complexos podem ser de difícil solução numérica.

No que diz respeito ao estudo da estabilidade a pequenos sinais de sistemas de energia elétrica, por muito tempo foram utilizados exclusivamente modelos que consistiam de uma única unidade geradora conectada a um barramento infinito. Embora esse modelo forneça resultados importantes para o entendimento de vários aspectos do comportamento de sistemas elétricos, com a interligação de várias unidades geradoras e de centros consumidores por meio de linhas de transmissão, tornou-se então necessário que os modelos utilizados passassem a representar de maneira mais precisa a topologia do sistema elétrico de potência (SEP).

Além da mudança na topologia, outro fator importante passou a ser considerado por uma série de pesquisadores em seus estudos: o comportamento da carga em face ao

1 andre.miyahara@yahoo.com

2 percival@dee.feis.unesp.br 
perfil de tensão e/ou frequência da rede elétrica [2-6].

Com o avanço da eletrônica de potência, um grande percentual das cargas conectadas ao SEP apresentam não linearidades. Essa mudança no perfil das cargas com os constantes avanços na tecnologia de medição e na implantação crescente das chamadas redes inteligentes, faz com que em um futuro não muito distante os modelos para estudo de sistemas de energia elétrica levem em conta esta nova característica das cargas bem como a consideração de outros fenômenos, como a presença de componentes harmônicas no SEP.

Neste trabalho cargas são representadas por um modelo exponencial dependente da magnitude da tensão e da frequência [2,3]. Tais cargas, agora não mais representadas por um modelo estático, são então incorporadas ao Modelo de Sensibilidade de Potência (MSP) [7] e avaliadas por simulações computacionais em um sistema teste.

\section{Formulação do Problema}

O MSP é um modelo para estudo da estabilidade a pequenas perturbações proposto em [7] e cuja formulação baseia-se na junção de equações diferenciais (que descrevem a dinâmica das máquinas síncronas conectadas ao SEP) com equações algébricas (obtidas a partir da aplicação do conceito do balanço nodal de potência em todas as barras do SEP). A aplicação do balanço nodal de potência à barra k permite obter (1).

$$
\begin{aligned}
P_{g_{k}} & =P_{L_{k}}+P_{\exp _{, k}} \\
Q_{g_{k}} & =Q_{L_{k}}+Q_{\exp _{, k}}
\end{aligned}
$$

Em (1), $\mathrm{P}_{\mathrm{g}}$ e $\mathrm{Q}_{\mathrm{g}}$ representam as potências ativa e reativa entregues pela geração à barra $\mathrm{k}$, enquanto que $\mathrm{P}_{\mathrm{L}} \mathrm{e} \mathrm{Q}_{\mathrm{L}}$ são as potências consumidas por cargas conectadas à barra $\mathrm{k}$. $\mathrm{P}_{\mathrm{exp}, \mathrm{k}} \mathrm{e}$ $\mathrm{Q}_{\text {exp,k }}$ representam as potências ativa e reativa totais que são exportadas a partir da barra $\mathrm{k}$ para as demais barras adjacentes. Admitindo-se pequenas perturbações em torno de um ponto de operação é possível linearizar estas equações pela expansão em série de Taylor, desconsiderando os termos de ordem maior que um. Após a linearização o balanço nodal de potência é dado por (2).

$$
\begin{aligned}
\Delta P_{g_{k}} & =\Delta P_{L_{k}}+\Delta P_{\exp _{, k}} \\
\Delta Q_{g_{k}} & =\Delta Q_{L_{k}}+\Delta Q_{\exp _{, k}}
\end{aligned}
$$

As expressões linearizadas referentes à potência gerada e à potência exportada da barra k para as demais barras adjacentes são dadas por (3) e (4), respectivamente [7].

$$
\begin{aligned}
& \Delta P_{g_{k}}=A 1_{g_{k}} \Delta \delta_{k}+A 2_{g_{k}} \Delta E_{q_{k}}^{\prime}+A 3_{g_{k}} \Delta v_{k}-A 1_{g_{k}} \Delta \theta_{k} \\
& \Delta Q_{g_{k}}=R 1_{g_{k}} \Delta \delta_{k}+R 2_{g_{k}} \Delta E_{q_{k}}^{\prime}+R 3_{g_{k}} \Delta v_{k}-R 1_{g_{k}} \Delta \theta_{k}
\end{aligned}
$$

Nas equações (3) e (4) $\delta_{k}$ é o ângulo interno da k-ésima máquina síncrona, $\theta_{k}$ é o ângulo da tensão da k-ésima barra do SEP, $E_{q k}^{\prime}$ é a tensão interna de eixo de quadratura da k-ésima máquina síncrona e $v_{k}$ é a magnitude da tensão da k-ésima barra do SEP. As constantes $A i_{g k}$ e $R i_{g k}$ são os coeficientes de sensibilidade de potência ativa e reativa associados à k-ésima máquina síncrona enquanto que $A i_{k, k}$ e $R i_{k, k}$ são os coeficientes de sensibilidade de potência ativa e reativa associados ao fluxo de potência exportado a partir da barra $\mathrm{k}$ para as barras adjacentes e relacionados com as variáveis da própria 
barra k. $A i_{k, i}$ e $R i_{k, i}$ são os coeficientes de sensibilidade de potência ativa e reativa associados ao fluxo de potência exportado a partir da barra $\mathrm{k}$ para as barras adjacentes e relacionados com as variáveis da barra vizinha i.

$$
\begin{gathered}
\Delta P_{\exp , k}=A 1_{k, k} \Delta \theta_{k}+\sum_{\substack{i=1 \\
i \neq k}}^{n}\left[A 1_{k, i} \Delta \theta_{i}\right]+A 2_{k, k} \Delta v_{k}+\sum_{\substack{i=1 \\
i \neq k}}^{n}\left[A 2_{k, i} \Delta v_{i}\right] \\
\Delta Q_{\exp , k}=R 1_{k, k} \Delta \theta_{k}+\sum_{\substack{i=1 \\
i \neq k}}^{n}\left[R 1_{k, i} \Delta \theta_{i}\right]+R 2_{k, k} \Delta v_{k}+\sum_{\substack{i=1 \\
i \neq k}}^{n}\left[R 2_{k, i} \Delta v_{i}\right]
\end{gathered}
$$

A potência consumida pelas cargas é representada pelo modelo exponencial apresentado em (5) [3,5] sendo que $P_{L k}^{0}$ e $Q^{0}{ }_{L k}$ são as potências ativa e reativa nominal das cargas, $v_{k}^{0}$ é a tensão nominal $(1 \mathrm{pu})$ e $\omega_{0}$ é a velocidade angular nominal (para a frequência de $60 \mathrm{~Hz}, \omega_{0}$ equivale a aproximadamente $377 \mathrm{rad} / \mathrm{s}$ ). $p_{v k}$ e $q_{v k}$ são constantes relacionadas à variação das potências ativa e reativa com o valor da magnitude da tensão na barra $\mathrm{k}$ e $p_{\omega k}$ e $q_{\omega k}$ são as constantes relacionadas à variação das potências ativa $\mathrm{e}$ reativa com o valor da frequência na barra $\mathrm{k}$.

$$
\begin{aligned}
& P_{L_{k}}=P_{L_{k}}^{0}\left(\frac{v_{k}}{v_{k}{ }^{0}}\right)^{p_{v k}}\left(\frac{\omega_{L_{k}}}{\omega_{0}}\right)^{p_{\omega k}} \\
& Q_{L_{k}}=Q_{L_{k}}^{0}\left(\frac{v_{k}}{v_{k}^{0}}\right)^{q_{v k}}\left(\frac{\omega_{L_{k}}}{\omega_{0}}\right)^{q_{\omega k}}
\end{aligned}
$$

A frequência em cada barra pode ser escrita em termos da variação angular de acordo com (6). Com isso a linearização de (5) resulta nas expressões dadas em (7).

$$
\begin{gathered}
\dot{\theta}_{k}=\omega_{L_{k}}-\omega_{0} \\
\Delta P_{L_{k}}=D 1_{k} \Delta v_{k}+D 2_{k} \Delta \dot{\theta}_{k} \\
\Delta Q_{L_{k}}=D 3_{k} \Delta v_{k}+D 4_{k} \Delta \dot{\theta}_{k}
\end{gathered}
$$

A dinâmica das máquinas síncronas responsáveis pela geração de potência elétrica é representada pelo conjunto de equações diferenciais (8) [7].

$$
\begin{gathered}
M_{k} \Delta \dot{\omega}_{k}=\Delta P_{m_{k}}-A 1_{g_{k}}\left(\Delta \delta_{k}-\Delta \theta_{k}\right)-A 2_{g_{k}} \Delta E_{q_{k}}^{\prime}-A 3_{g_{k}} \Delta v_{k}-D_{k} \Delta \omega_{k} \\
\Delta \dot{\delta}_{k}=\omega_{0} \Delta \omega_{k} \\
T_{d 0_{k}}^{\prime} \Delta \dot{E}_{q_{k}}^{\prime}=\Delta E_{f d_{k}}-\frac{x_{d_{k}}}{x_{d_{k}}^{\prime}} \Delta E_{q_{k}}^{\prime}+K_{v_{k}} \Delta v_{k}-K_{A_{k}}\left(\Delta \delta_{k}-\Delta \theta_{k}\right) \\
T_{r_{k}} \Delta \dot{E}_{f d_{k}}=K_{r_{k}}\left(\Delta v_{r e f_{k}}-\Delta v_{k}\right)-\Delta E_{f d_{k}}
\end{gathered}
$$

No conjunto (8) $M_{k}$ é constante de inércia, $\omega_{k}$ é a velocidade angular do rotor, $P_{m k}$ é a potência mecânica de entrada, $D_{k}$ é o amortecimento inerente do SEP. O sistema de excitação acoplado ao gerador $\mathrm{k}$ é representado por um bloco de primeira ordem de ganho $k_{r k}$ e constante de tempo $T_{r k}$. A tensão de campo do gerador é representada por $E_{f d k}$ e $T_{d o k}^{\prime}$ é a constante de tempo transitória de circuito aberto de eixo direto. A reação de 
armadura da máquina síncrona é representada pelas constantes $k_{v k} \mathrm{e} k_{a k}$.

As equações diferenciais (8) em conjunto com as equações algébricas (2) constituem o MSP. Cabe a ressalva de que com a inclusão de cargas cujas demandas de potência sejam funções da frequência de suas respectivas barras, há um aumento na quantidade de variáveis de estado e uma redução de mesma ordem na quantidade de variáveis algébricas. Isto ocorre porque a variação do ângulo da tensão nas barras em que tais cargas se encontram passa a ser uma variável de estado.

\section{Métodos e Resultados}

Para analisar a influência da consideração de cargas cuja potência é função da tensão e da frequência tal como em (5) foram realizadas simulações utilizando-se o software MATLAB $^{\circledR}$. O sistema teste avaliado é conhecido como New England, cujo diagrama unifilar é dado na Figura 1. Este SEP é formado por 10 geradores separados em duas áreas, com um total de 39 barras, sendo que em 19 delas estão conectadas cargas (dados do ponto de operação considerados podem ser encontrados em [8]). A representação do sistema New England pelo MSP sem que sejam considerados modelos dinâmicos para representar as cargas (caso base) resulta em um total de 22 autovalores reais e negativos e 9 pares de autovalores complexos conjugados, mostrados na Tabela 1.

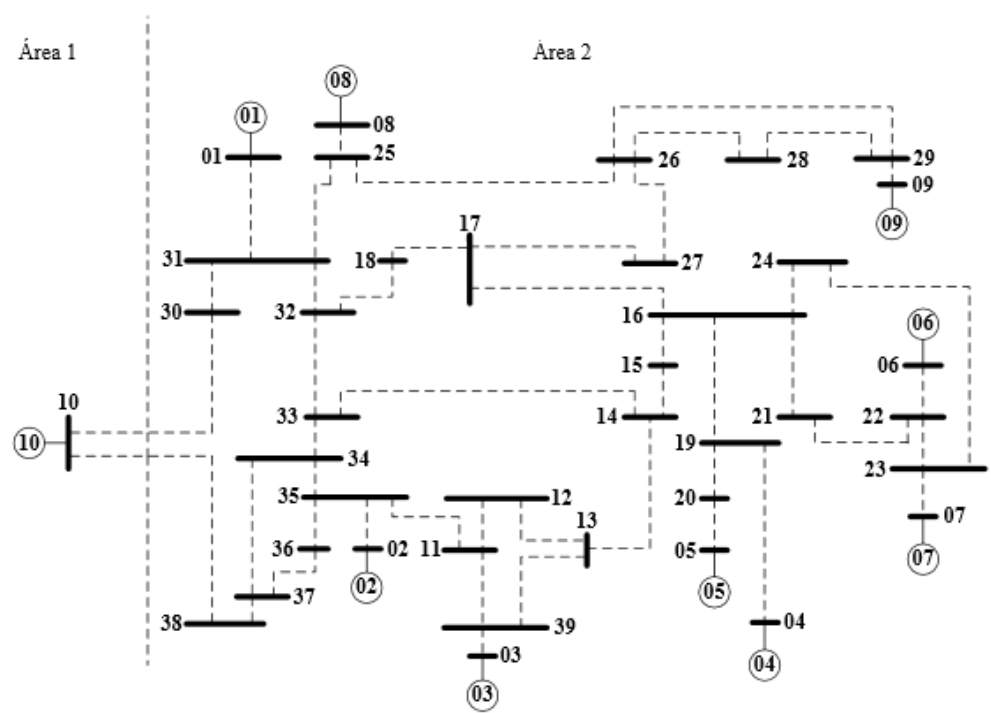

Figura 1: Diagrama unifilar do sistema New England

Analisando estes autovalores conclui-se que o sistema teste é instável para o ponto de operação considerado devido à falta de amortecimento do modo de oscilação interárea e de 5 dos 8 modos de oscilação locais.

$\mathrm{Na}$ sequência, são feitas simulações em que apenas uma das 19 cargas do sistema é representada através do modelo representado por (5). Em todos os casos foram adotados $p_{v}=$ $q_{v}=0 ; p_{\omega}=0,6$ e $q_{\omega}=-2,5$. Para todas as 19 simulações verificou-se que a representação das 
cargas através de um modelo dinâmico resultou no surgimento de um novo par de autovalores complexos conjugados, além de um aumento na quantidade total de autovalores do modelo, passando de 40 para 41 . Os autovalores de alguns desses casos se encontram apresentados na Tabela 1.

Tabela 1: Autovalores Dominantes: Sistema New England

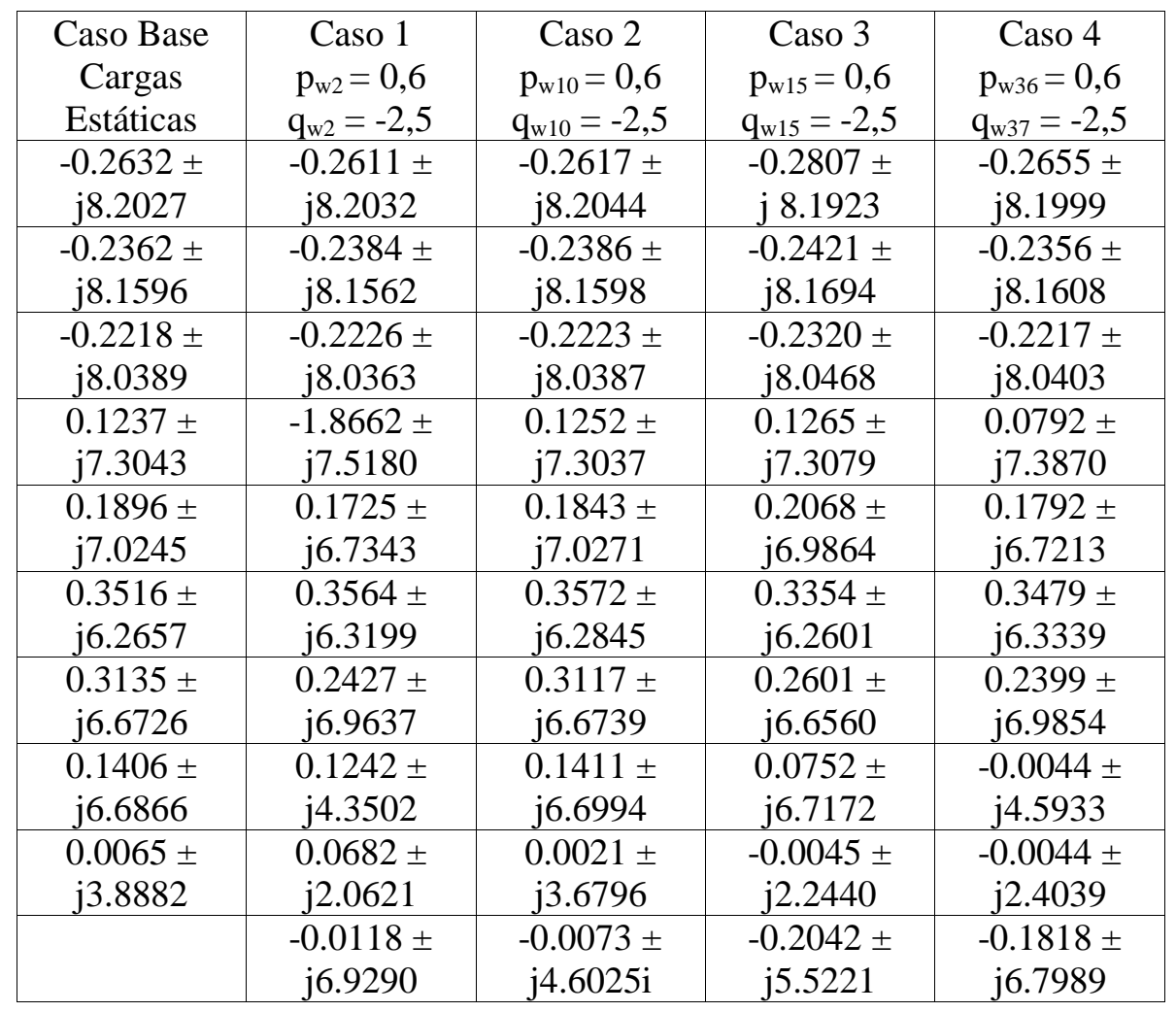

\section{Conclusões}

Os resultados obtidos mostram que, se apenas uma pequena parte das cargas for modelada adequadamente através do modelo exponencial, os resultados numéricos tendem a serem semelhantes aos obtidos com a representação das cargas por modelos estáticos. Dessa forma, fica evidente que a consideração da dinâmica das cargas se toma um fator que não pode ser desprezado apenas se uma grande parcela das cargas do sistema for representada de tal maneira.

Deve-se atentar entretanto que, a representação errônea das características dinâmicas das cargas pode levar o modelo a fornecer conclusões incorretas. Como exemplo desse fato, tem-se que ao simular uma carga cujo fator de potência sugere que os coeficientes relacionados a frequência sejam bem distintos de 0,6 e -2,5 verifica-se que o modelo passa a fornecer um autovalor real e positivo. Logo, nota-se que o grande desafio dessa área de estudo se encontra na obtenção de uma representação adequada das características das cargas conectadas ao sistema elétrico.

Com a expansão das chamadas redes inteligentes ou smart grids, o mapeamento da característica e do 
comportamento das cargas em face às variações dinâmicas da tensão e da frequência do sistema poderá fomecer dados valiosos para a formulação e uso de novos modelos dinâmicos que poderão ser incorporados nos modelos existentes para estudo da estabilidade a pequenas perturbações. Assim, sugere-se para trabalhos futuros que sejam abordados temas relacionadas à modelagem das cargas do sistema elétrico permitindo que a dinâmica das cargas seja analisada de maneira mais precisa.

\section{Agradecimentos}

Os autores agradecem à Coordenação de Aperfeiçoamento de Pessoal de Nível Superior pelo suporte financeiro à pesquisa.

\section{Referências}

[1] P. M. Anderson and A. A. Fouad, Power System Control and Stability, Iowa State University Press, (1977).

[2] T. S. Bhatti and D. J. Hill, A multimachine Heffron-Phillips model for power systems with frequency- and voltage-dependent loads, International Journal of Electric Power \& Energy Systems, vol. 12, 171-182, (1990), DOI: 10.1016/0142-0615(90)90030-F

[3] C. J. Berg, Power System Load Representation, Proceedings of IEEE, vol. 120, $344-$ 348, (1973), DOI: 10.1049/piee.1973.0071

[4] A. R. Bergen and D. J. Hill, A structure preserving model for power system stability analysis, IEEE Transactions on Power Apparatus and Systems, vol. PAS-100, 25-35, (1981), DOI: 10.1109/TPAS.1981.316883

[5] M. Brucoli, M. La Scala, R. Sbrizzai and M. Trovato, Voltage stability analysis of electric power systems with frequency dependent loads, IEE Proceedings C: Generation, Transmission and Distribution, vol. 140, 1-6, (1993).

[6] C. Concordia and S. Ihara, Load representation in power system stability studies, IEEE Transactions on Power Apparatus and Systems, vol. PAS-101, 969-977, (1982), DOI: 10.1109/TPAS.1982.317163.

[7] S. M. Deckmann e V. F. Costa, A Power Sensitivity Model for Electromechanical Oscillation Studies, IEEE Transactions on Power Systems, vol. 9, n. 2, 965-971, (1994), DOI: $10.1109 / 59.317649$.

[8] C. R. Pádua Jr., Modelos Lineares de Sistemas Elétricos de Potência: um estudo comparativo, Dissertação de Mestrado em Automação, UNESP, (2014). 